\title{
BMJ Open Effect of 12-week pulmonary rehabilitation on cognitive function in patients with stable chronic obstructive pulmonary disease: study protocol for a single-center randomised controlled trial
}

\author{
Hongxia Duan (D) , ${ }^{1}$ Peijun Li, ${ }^{2}$ Zhenwei Wang, ${ }^{3}$ Haixia Chen, ${ }^{2}$ Ting Wang, ${ }^{2}$ \\ Weibing $\mathrm{Wu},{ }^{2}$ Xiaodan Liu (i) ${ }^{1}$
}

To cite: Duan H, Li P, Wang Z, et al. Effect of 12-week pulmonary rehabilitation on cognitive function in patients with stable chronic obstructive pulmonary disease: study protocol for a single-center randomised controlled trial. BMJ Open 2020;10:e037307. doi:10.1136/ bmjopen-2020-037307

- Prepublication history and additional material for this paper is available online. To view these files, please visit the journal online (http://dx.doi.org/10. 1136/bmjopen-2020-037307).

HD and PL contributed equally.

Received 04 February 2020 Revised 23 August 2020 Accepted 06 September 2020

Check for updates

(c) Author(s) (or their employer(s)) 2020. Re-use permitted under CC BY-NC. No commercial re-use. See rights and permissions. Published by BMJ.

For numbered affiliations see end of article.

Correspondence to

Dr Xiaodan Liu;

hzhp403@126.com

\section{ABSTRACT}

Introduction Cognitive impairment, an important complication in patients with chronic obstructive pulmonary disease (COPD), seriously affects selfmanagement of the disease and quality of life $(Q 0 \mathrm{~L})$. As an exercise-based intervention programme, pulmonary rehabilitation (PR) — especially aerobic exercise (mainly mind-body exercise) and resistance exercise (RE) — has been proposed for its potential effectiveness in improving cognitive function. However, there is still a lack of strong evidence for PR's effectiveness. In this study, we expect to clarify the effects of pulmonary-based Qigong exercise and elastic band-based RE on cognitive function in patients with COPD and to fill in the relevant evidence blanks. Methods and analysis This study is a single-centre randomised controlled trial with assessor and data analyst blinding. We will recruit 108 participants with stable COPD starting on 23 December 2019, and randomly allocate them into the pulmonary-based Qigong exercise group, elastic band-based RE group, pulmonary-based Qigong exercise and elastic band-based RE combined group, or control group at a 1:1:1:1 ratio. Participants in intervention groups will perform 30 min of exercise two times per day, 5 days a week, for 12 weeks. The primary outcome will be the global cognitive function as assessed by the Montreal Cognitive Assessment and auditory event-related potential P300. Secondary outcomes will include the specific cognitive domains-attention, memory, executive function, verbal fluency and mental-processing speed; psychological functions and QoL. Exploratory outcomes will include grey matter volume and levels of inflammatory mediators. Outcomes will be measured before and after the interventions.

Ethics and dissemination Ethics approval has been granted by the Ethics Committee of Yue-Yang Integrative Medicine Hospital, an affiliate of Shanghai University of Traditional Chinese Medicine, Shanghai, China (Grant No. 2019-141). Written informed consent will be obtained from each participant before any procedures are performed. The findings will be published in peer-reviewed journals and presented at academic conferences.

\section{Strengths and limitations of this study}

- Compare and contrast the feasibility and outcomes following three exercise interventions using a robust randomised controlled trial design and methodology, and include a multidimensional assessment of cognitive function in patients with chronic obstructive pulmonary disease covering global cognitive function (GCF), attention, memory, executive function, verbal fluency and mental-processing speed, and grey matter volume.

- In this study, the main interventions do not require costly specific instruments or much space and can therefore be used for patients who exercise in the community or at home.

- This study combines the Montreal Cognitive Assessment with auditory event-related potential P300 to objectively and precisely assess GCF.

- Assessment results might be affected by subjective bias from the participants in this study because it would be impossible to blind them to exercise as an intervention.

- The effective resistance of the elastic bands cannot be accurately measured during the exercise, and we will strictly control and calibrate the actions of the participants through face-to-face teaching and video learning to achieve the purpose of effective resistance.

Trial registration number ChiCTR1900026869; preresults.

\section{INTRODUCTION}

Chronic obstructive pulmonary disease (COPD) is a systemic disease that impairs physiology, psychology and cognition. ${ }^{1}$ Multiple studies with different sample sizes and cognitive-function assessment tools have determined that the prevalence of 
cognitive impairment $(\mathrm{CI})$ in patients with COPD ranges from $17 \%$ to $56.7 \%$, which is significantly higher than that in healthy controls $(12 \%-12.7 \%){ }^{2}$ CI in patients with COPD is mainly manifested in attention function, memory function, executive function, verbal fluency and mental-processing speed, ${ }^{3-6}$ which affect physical performance, psychological functions and quality of life (QoL). ${ }^{78}$ However, relevant research on the treatment of $\mathrm{CI}$ in patients with COPD is rare.

Pulmonary rehabilitation (PR), including but not limited to exercise, health education and behaviour change training, is a comprehensive, multidisciplinary, non-pharmacological treatment based on medical assessment results of patients with COPD. ${ }^{9}$ Aerobic exercise (AE) or resistance exercise (RE) at moderate intensity, combined with breathing, is conducive to COPD recovery and plays a positive role in improving cognitive function in patients with COPD. ${ }^{10}{ }^{11} \mathrm{In} \mathrm{AE}$, mind-body exercises such as traditional Chinese exercises (TCEs) can significantly improve the QoL of patients with COPD compared with walking training. ${ }^{12}$ Our previous studies also found that TCEs significantly improved lung function, exercise capacity and QoL in patients with COPD. ${ }^{13} 14$ Moreover, a study that applied 6-month Baduanjin for patients with COPD found significant improvements in global cognitive function (GCF; measured by Mini-Mental State Examination (MMSE), as well as by P300), psychological functions (measured by the Hamilton Anxiety Scale (HAMA), and the Hamilton Depression Scale (HAMD)), and QoL. ${ }^{15}$ These findings suggest that TCEs have a positive effect on GCF in patients with COPD. TCEs, including Liuzijue, Baduanjin, Wuqinxi and Yijinjing, were organized by the General Administration of Sport of China on the basis of ancient Chinese medicine books in 2003 and are popularised nationwide. They have different characteristics, but only some of them are focused on improving lung function. Therefore, this study rearranged TCEs in order to improve cognitive function in patients with COPD from the perspective of improving lung function; we refer to the new form as pulmonary-based Qigong exercise. The movements include the 'HU' and 'SI' exercises in Liuzijue; 'pushing up the sky to regulate the Triple Warmer (meridian)' and 'drawing a bow to shoot a vulture' in Baduanjin; 'the crane actions, including the crane spreading its wings and the crane flying' in Wuqinxi; and 'cross-armed iron staff' in Yijinjing. ${ }^{16-18}$

$\mathrm{RE}$, as a load-training method targeted at specific muscles, can improve selective attention, associative memory and functional plasticity of the brain in the elderly. ${ }^{19}$ Elastic band-based RE can enhance GCF and delay decline in cognitive function in the elderly. ${ }^{20}{ }^{21}$ The above mentioned results imply that the advantages of RE in CI for patients with COPD are worth exploring. Also, a previous study has shown that a 4-week $\mathrm{AE}$ and $\mathrm{RE}$ combination regimen significantly improved long-term memory, apraxia and logical-reasoning ability compared with $\mathrm{AE}$ alone. ${ }^{22}$ Another study compared the effects of $\mathrm{AE}$ and $\mathrm{RE}$ combination regimen with those of $\mathrm{RE}$ alone on executive function and attention in men and found similar results. ${ }^{23}$ However, a third study found no difference between $\mathrm{AE}, \mathrm{RE}$, and $\mathrm{AE}$ combined $\mathrm{RE}$ after 12 weeks in improving GCF (as measured by the Montreal Cognitive Assessment (MoCA)) in the elderly. ${ }^{24}$ Therefore, it is necessary to explore the advantages of $\mathrm{AE}$ and $\mathrm{RE}$ combination regimen on cognitive function in patients with COPD.

The main purpose of this study is to explore the effects of 12-week regimens of pulmonary-based Qigong exercise, elastic band-based RE, and the combination of both exercise interventions on GCF, specific cognitive domains (attention, memory, executive function, verbal fluency and mental-processing speed), psychological functions, and QoL in patients with stable COPD. Its secondary purpose is to explore the possible mechanism by which exercise intervention improves cognitive function in COPD, based on brain grey matter volume (GMV) and levels of blood inflammatory mediators. We hypothesised that all three PR regimens are positive significantly, and that combined exercise emerges additional benefits in patients with COPD compared with single exercise methods.

\section{METHODS}

\section{Design}

This is a single-center randomised controlled trial with assessor and data analyst blinding. Participants will be enrolled at the Respiratory Medicine Department of Yue-Yang Integrative Medicine Hospital, an affiliate of Shanghai University of Traditional Chinese Medicine. Participants will be randomly assigned to the pulmonarybased Qigong exercise group (QG), elastic band-based $\mathrm{RE}$ group (RG), pulmonary-based Qigong exercise and elastic band-based RE combined group (QRG), or control group at a 1:1:1:1 ratio. All participants will receive usual medical care and health education three times for 12 weeks; those in the intervention groups will receive pulmonary-based Qigong exercise, elastic band-based RE or a combination of both exercise. The primary outcome will be GCF as assessed by MoCA and auditory event-related potential (AERP) P300. Secondary outcomes will include the specific cognitive domains as assessed separately by the attention subtest (AT) of the MoCA, Rivermead Behavioural Memory Test-II (RBMTII), Trail Making Tests Part A and B (TMT-A, TMT-B), Verbal Fluency Test (VFT) and Digit Symbol-Coding Test (DSC) in Wechsler Adult Intelligence Scale (WAIS); psychological functions as assessed by HAMA and HAMD; QoL as assessed by St. George's Respiratory Questionnaire (SGRQ). Exploratory outcomes will include GMV and levels of inflammatory mediators. Outcomes will be measured within 1 week before and after the interventions. A flow diagram of the study design is shown in figure 1 . The study protocol strictly follows the Standard Protocol Items: Recommendations for Interventional 


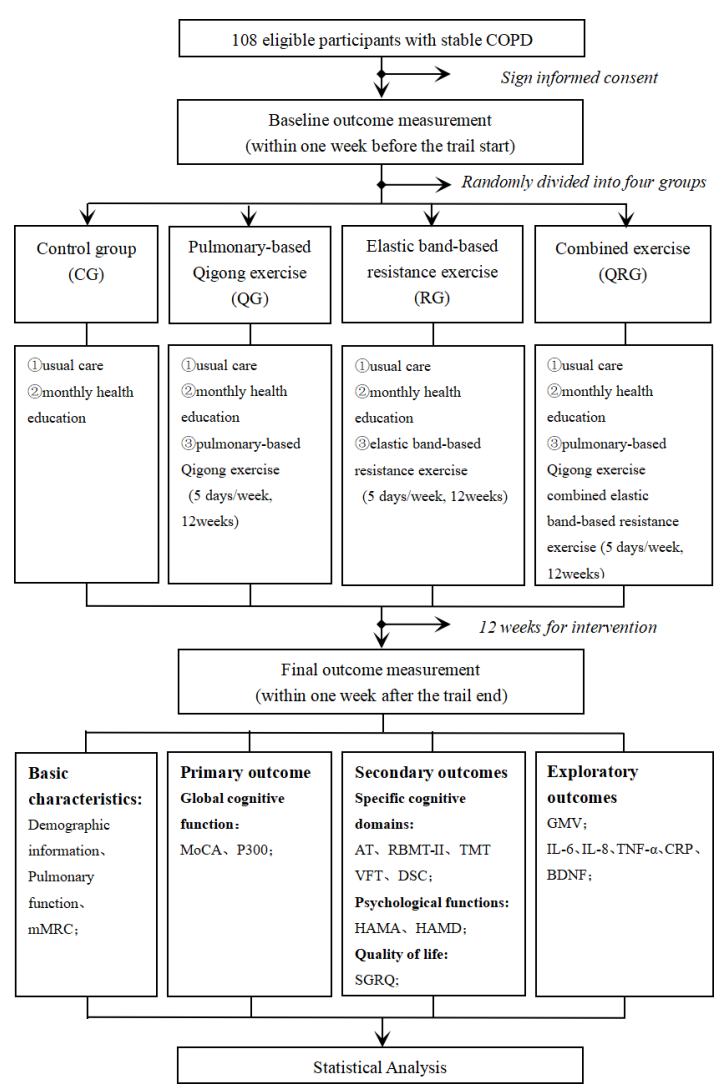

Figure 1 A flow diagram of the study design. AT, attention subtest; BDNF, brain-derived neurotrophic factor; COPD, chronic obstructive pulmonary disease; CRP, $\mathrm{C}$ reactive protein; DSC, Digit Symbol Coding; GMV, grey matter volume; HAMA, Hamilton Anxiety Scale; HAMD, Hamilton Depression Scale; IL, interleukin; mMRC, modified Medical Research Council Dyspnoea Scale; MoCA, Montreal Cognitive Assessment; RBMT-II, Rivermead Behavioural Memory Test-II; SGRQ, St. George's Respiratory Questionnaire; TMT, Trail Making Test; TNF- $\alpha$, tumour necrosis factor- $\alpha$; VFT, Verbal Fluency Test.

Trials (SPIRIT) checklist (online supplemental file 1) and SPIRIT figure (online supplemental file 2).

\section{Patient and public involvement}

No patients and/or public has been involved in the design, or conduct, or reporting, or dissemination plans of our research.

\section{Sample size}

This is a superiority trial. The sample size is calculated based on the MoCA score using the PASS V.11.0 software. The means and SDs of MoCA in the control and AE and RE combined group were $21.20 \pm 2.11$ and $24.60 \pm 2.26$, respectively. ${ }^{25}$ Setting alpha $=0.025$ (one-sided), power $=0.90$, superiority margin $=1.13$, we calculated the minimum sample size of 21 participants per group. Furthermore, we conducted a power analysis to estimate the sample size needed to perform four group comparisons provided that an $\alpha$ is set as $0.008 .{ }^{25-27}$ The results showed that recruitment of 27 participants would achieve a statistical power of 0.99 . Considering a $20 \%$ attrition rate, a total of 108 participants with 27 participants per group is a reasonable sample size for this study.

\section{Participants}

Inclusion criteria

Participants will be included if they (1) are diagnosed with COPD according to the 2019 Global Initiative for Chronic Obstructive Lung Disease criteria; (2) are clinically stable in the 4 weeks before randomisation (confirmed by absence of acute exacerbation and related drug changes); (3) are 40-80 years of age and (4) are willing to give written informed consent.

\section{Exclusion criteria}

Exclusion criteria are as follows: (1) coexistence with other respiratory diseases, including asthma, tuberculosis, bronchiectasis and lung cancer; (2) have been suffering from a severe medical condition that might affect exercise capacity, such as the heart, brain, blood vessels, liver, kidneys, endocrine system, nervous system or musculoskeletal system that might affect exercise capacity; (3) participation in regular exercise training (at least two times per week) in the past 6 months; (4) have been suffering from brain tumour, brain trauma or other diseases that could cause cognitive impairment or (5) presence of a severe medical condition that makes the individual unable to follow instructions properly.

\section{Recruitment and screening}

The source of recruitment is the Respiratory Medicine Department of Yue-Yang Integrative Medicine Hospital, where a large number of patients guarantee that enough participants can be recruited. The recruitment will begin on 23 December 2019. The recruitment procedures are as follows: First, the primary researchers will introduce the purpose and the inclusion and exclusion criteria of this study to the recruiting researchers. Then, the recruiting researchers will introduce the purpose, content, and possible risks and benefits of this study to the subjects who have been diagnosed with COPD. If the subjects are interested in participating in the study, the recruiting researchers will talk with subjects in detail to confirm whether they meet the inclusion and exclusion criteria. Finally, the eligible potential subjects or their families will provide signed informed consent to protect their privacy.

\section{Randomisation and blinding}

To ensure that the risk of bias remains low, participants will be randomly allocated to one of four groups by an independent person not participating in any other aspect of this study, according to a random number table generated by SPSS V.24.0. Random numbers will be placed in opaque envelopes to achieve allocation concealment, and only the primary researchers will know the randomly allocated sequence. In addition, the assessor and data analyst will be blinded, and they will be replaced once they know the allocation. The new assessor will continue to carry out the assessment, and the new data analyst will re-analyse 


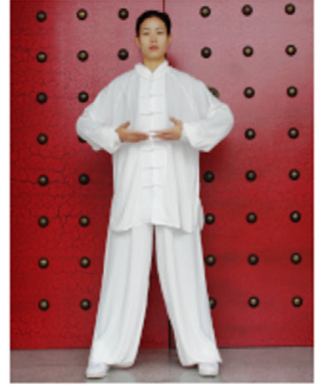

A "starting posture"

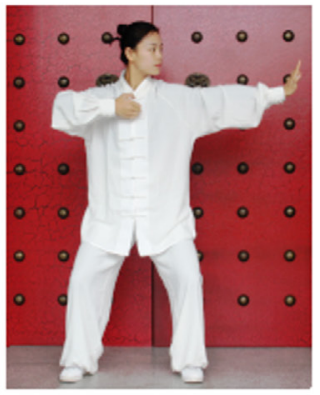

E "drawing a bow

to shoot a vulture"

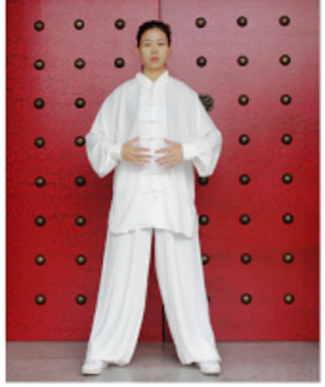

B "HU exercise"

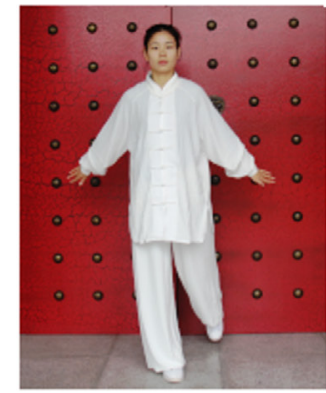

F "the crane actions"

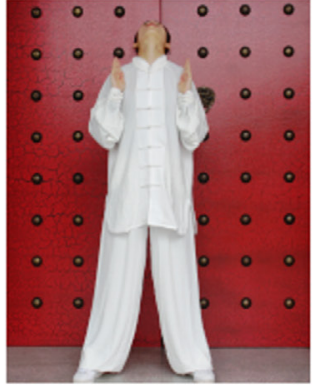

C "SI exercise"

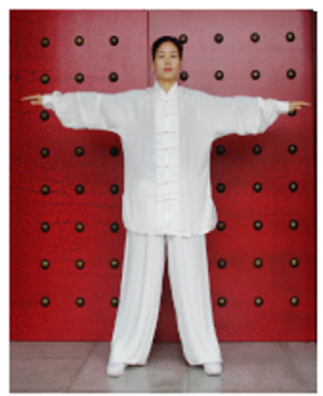

G "cross-armed iron staff'

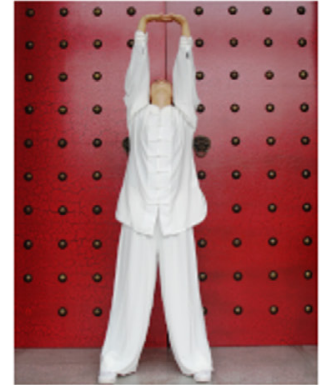

D "pushing up

the sky to regulate
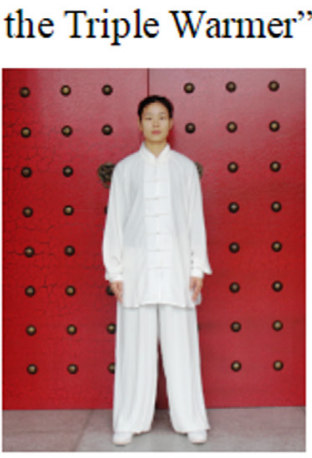

$\mathrm{H}$ "ending posture"

Figure 2 The movements of pulmonary-based Qigong exercise. The participant in the figure is Haixia Chen in the author list.

all data. Meanwhile, we will conduct standard teaching to the assessor through unified learning and practical training within 1 week before the initial and the final assessment, and participants will be strictly required to not discuss interventions with the assessor.

\section{Intervention}

All participants will receive usual medical care and monthly health education sessions. Each health education session will last for $60 \mathrm{~min}$ and cover the concept, risk factors and self-management of COPD. The control group will be told to maintain their extant daily habits, while the intervention group will be instructed by professional physiotherapists to master specific exercises 1 week before the formal intervention. Interventions will be conducted two times per day (morning and afternoon) for $30 \mathrm{~min}, 5$ days a week. During 1 of these 5 days, participants will gather at the hospital to receive intervention and get feedback; they will exercise at home for the remaining 4 days. The training load will be monitored using Borg CR10 scale at a level of 4-6. ${ }^{1}$ If one always cannot perform the specific exercise at the right intensity, then just perform at bestachieved intensity. And the researchers will record it.

\section{Pulmonary-based Qigong exercise group (QG)}

Participants in the QG will receive a 12-week intervention of pulmonary-based Qigong exercise (figure 2). If the Borg reported is below 4 or over 6 , participants will be instructed to increase/decrease their intensity, which includes changing the range of motion, speed and breathing method.

\section{Elastic band-based RE group (RG)}

Participants in the RG will receive a 12-week intervention of elastic band-based RE. We will use TheraBand elastic bands (The Hygenic Corporation, Akron, Ohio, USA) to provide additional resistance for progressive-RE. Different colours indicate different resistance: brown $<$ yellow $<$ red $<$ green $<$ blue $<$ black $<$ silver $<$ gold. The resistance difference between two adjacent colours is $20 \%-30 \%,{ }^{27}$ which will allow a suitable level of resistance for each participant.

In the elastic band-based RE regimen, four upper-limb resistance movements performed by proximal muscles have been selected to improve breathing, and four lowerlimb resistance movements to improve walking (figure 3). Before the formal intervention, one repetition maximum $(1 \mathrm{RM})$ that the participant can bear in one exercise will be obtained via the submaximal test $(10 \mathrm{RM}=75 \% 1 \mathrm{RM}) .{ }^{28}$ The equipment for the 10RM test is the TheraBand elastic band (based on the TheraBand force-elongation table), ${ }^{29}$ and the exercises in the test are eight resistance movements in the elastic band-based RE regimen. Referring to similar literature and making appropriate adjustments, exercise intensity will be $30 \%-40 \% 1 \mathrm{RM}$ in the 1 st month, $40 \%-50 \% 1 \mathrm{RM}$ in the 2nd month and 50\%-60\% $1 \mathrm{RM}$ in the 3 rd month. ${ }^{30}$ Each session will be composed of 3 sets of 8-12 repetitions of each exercise, with a rest period 

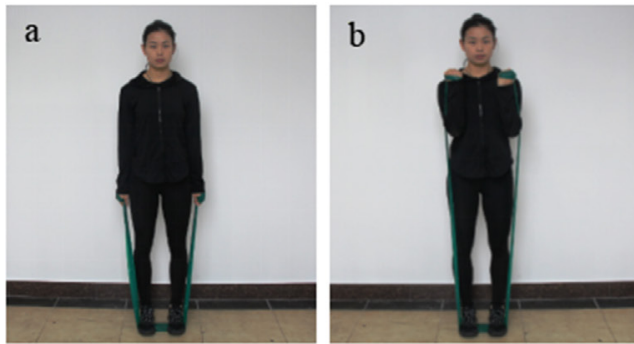

A "elbow flexion"
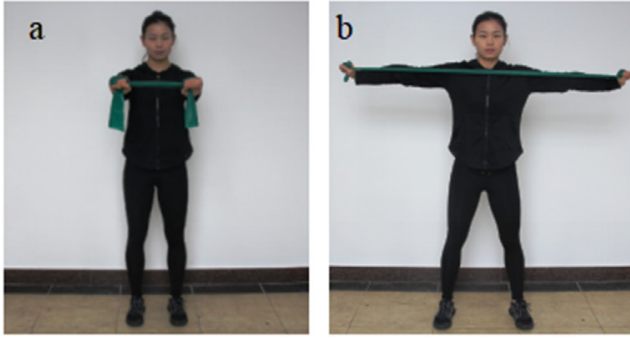

C "shoulder horizontal abduction"
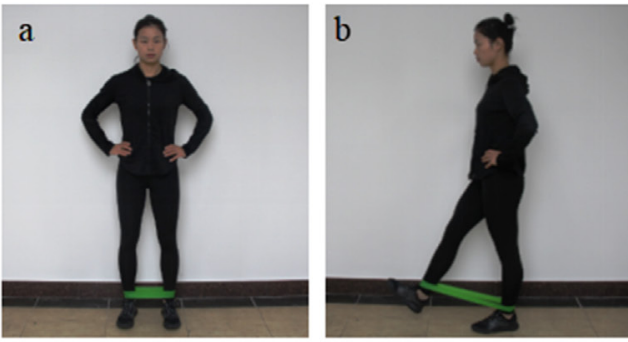

E "hip flexion"
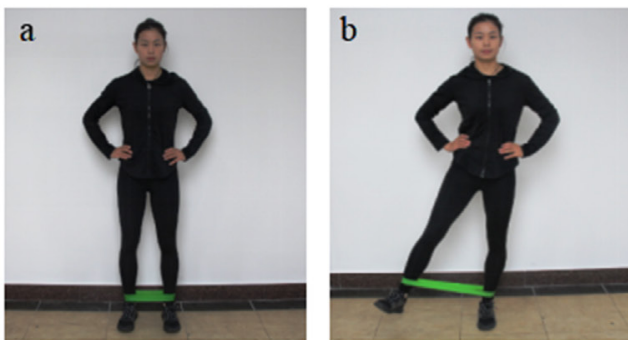

G "hip abduction"
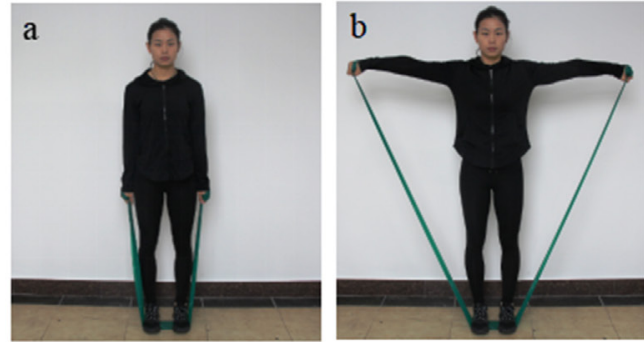

B "shoulder abduction"
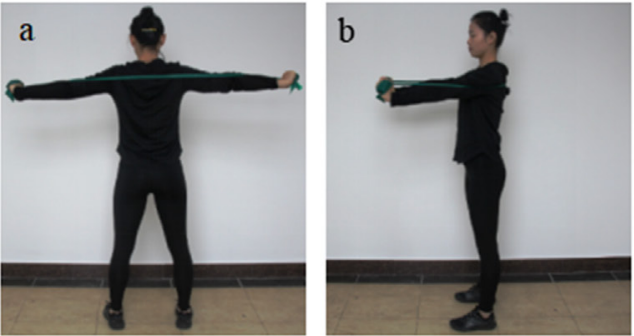

D "shoulder horizontal adduction"

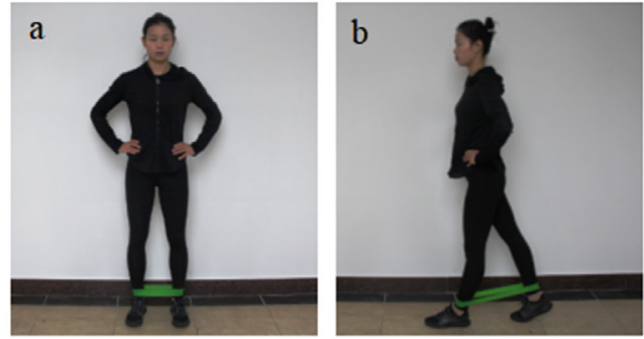

F "hip extension"
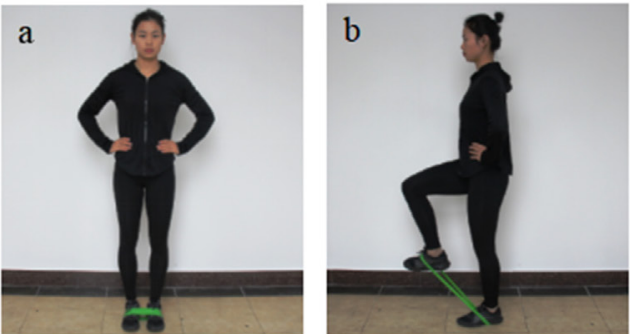

$\mathrm{H}$ "hip flexion and knee flexion"

Figure 3 The movements of elastic band-based resistance exercise: ' $a$ ' is the starting position and ' $b$ ' is the end position. The participant in the figure is Haixia Chen in the author list.

of 1-3 min between sets. ${ }^{31}$ If the Borg reported is below 4 or over 6 , participants will be instructed to increase/ decrease their intensity, which includes changing the colour of the elastic band (including add extra elastic band), repetitions and speed.

\section{Pulmonary-based Qigong exercise and elastic band-based} resistance exercise combined group group (QRG)

Participants in the QRG will receive a 12-week intervention of pulmonary-based Qigong exercise combined with elastic band-based RE. Total duration of intervention is the same as QG and RG. Participants in this group will be training pulmonary-based exercise for $30 \mathrm{~min}$ and elastic band-based RE for 30 min every day.

\section{Outcome measurement}

Basic characteristics

\section{Pulmonary function}

Specialists will perform the pulmonary-function test using a spirometer (MasterScreen PFT; Jaeger Toennies Erich Jaeger GmbH, Höchberg, Germany) and referring to American Thoracic Society/European Respiratory Society guidelines. ${ }^{32}$ Each participant will be tested three times, and the final result used in the analysis will be the best value.

\section{Dyspnoea symptoms}

The subjective severity of dyspnoea in participants will be measured using the modified Medical Research Council Dyspnoea Scale (mMRC). ${ }^{1}$ The $\mathrm{mMRC}$ is a 5 -point scale 
$(0-4)$ on which higher scores indicate worse levels of dyspnoea.

\section{Primary outcome}

GCF will be measured using MoCA and AERP P300. The MoCA covers 8 aspects of cognitive areas: visual space and execution, naming, memory, attention, language, abstraction, delayed recall and orientation. ${ }^{33} \mathrm{~A}$ total score of 30 points and a score of $\geq 26$ points indicate no impairment of cognitive performance. The P300 will be recorded using a 64-channel electroencephalogram signal acquisition recorder (BrainCap MEG; Brain Products, GmbH, Gilching, Germany). A total of 300 stimuli will be presented at $80-85 \mathrm{~dB}$ according to the 'oddball' paradigm. The 'oddball' (novelty/rare) tone will be set at $2 \mathrm{kHz}$, accounting for $20 \%$, while the standard tone will be set at $1 \mathrm{kHz}$, accounting for $80 \%$. The bandpass filter will be set in the range of $0.1-50 \mathrm{~Hz}$. Participants will be required to count instances of the 'oddball' tone. Brain Vision Analyzer software V.2.2.0 (Brain Products) will be used for the final analysis of the signals collected. ${ }^{345}$

\section{Secondary outcomes}

Secondary outcomes include the specific cognitive domains (ie, attention, memory, executive function, verbal fluency and mental-processing speed), psychological functions and QoL.

- Attention function will be assessed using the AT of the MoCA. This test includes three items, with a total score of 7 points. Higher scores indicate better attention. ${ }^{36}$

- Memory function will be assessed using the RBMT-II that contains 13 items. ${ }^{37}$ Two parallel versions of the RBMT-II with the same test modes but different materials will be used at the start and end of the intervention. Total score is 24; lower scores indicate greater impairment.

- Executive function will be assessed using the TMT, including the TMT-A and TMT-B. Part A requires participants to connect 25 numbers in order; Part B requires them to alternately connect 25 numbers in squares and circles in order. ${ }^{38}$ Completion time will be recorded.

- Verbal fluency will be assessed using the VFT, which requires participants to speak the names of animals, vegetables or fruits aloud as often as possible within 60 s. ${ }^{39}$ The number of the names spoken will be recorded.

- Processing speed will be assessed using the DSC subtest of the WAIS. ${ }^{40}$ Participants will be required to turn the numbers in the list into corresponding symbols as quickly as possible from left to right within 90 s. Each correct symbol will be scored 1 point; an inverted symbol will be scored 0.5 points. Maximum score is 90 points.

- Psychological functions will be measured using HAMA and HAMD that participants will complete via self-assessments. HAMA will be used to assess anxiety symptoms, which includes 24 items and rated 0-4. HAMD will be used to assess depressive symptoms, which includes 24 items and rated $0-2$ or $0-4$. The higher the score, the more severe the degree of anxiety and depression. ${ }^{41-43}$

- QoL will be measured using SGRQ that is a COPDspecific assessment tool. ${ }^{43}$ The SGRQ contains 50 items, covering symptoms, activities and impacts. The score ranges from 0 to 100,0 indicating no impairment, and 100 indicating extreme impairment.

\section{Exploratory outcomes}

\section{Grey matter volume}

Specialists will test GMV using a Magnetom Verio 3.0 Tesla MRI system (Siemens, Munich, Germany). MRI is a non-invasive method of examining brain activity and structure. ${ }^{44}$ We will use T1 weighted-image 3-dimensional magnetisation-prepared rapid-acquisition gradient-echo sequence to collect T1-weighted structural images.

\section{Inflammatory mediators}

The inflammatory mediators including interleukin- 6 and 8 (IL-6, IL-8), tumour necrosis factor- $\alpha$ (TNF- $\alpha$ ), C reactive protein (CRP) and brain-derived neurotrophic factor (BDNF) will be assayed. Plasma and serum samples will be stored at $-80^{\circ} \mathrm{C}$ as required.

\section{Monitoring of participant compliance}

Physical activity and energy expenditure of participants will be assessed using a triaxial accelerometer (ActiGraph GT3X+; Actigraph LLC, Pensacola, Florida, USA) at the start and end of intervention. Participants will be instructed to carry the accelerometer on an elastic belt on the right side of the hip for 7 consecutive days; they will be allowed to remove it during sleeping hours and personal grooming. The threshold of valid data will be set at 10 hours/day. During the test, participants will be required to record the time and reason for removing the accelerometer.

\section{Safety}

During the trial, any adverse events will be recorded in a timely manner and reported to the primary researchers, who will then decide whether these adverse events are related to the trial. When a serious adverse event occurs, the Ethics Committee and a clinician from the Department of Respiratory Medicine of Yue-Yang Integrative Medicine Hospital will be informed so that they can provide treatment in a timely manner. For patients who cannot continue training for other reasons, the researchers must record the reason for the drop-out in the case report form, keep in touch with the subjects and complete the assessment as much as possible, and fill out the trial end form.

\section{Monitoring of research quality}

Compliance of participants in the control group will be judged based on whether they change drugs due to disease or whether they participated in other regular physical exercises in the preceding 12 weeks. Compliance of participants in the intervention groups will be monitored 
using the exercise recording cards collected during the weekly intensive exercise. Participants whose actual exercise sessions account for $>85 \%$ of the estimated number of exercise sessions will be judged as having received a valid intervention.

\section{Data management}

All medical records and assessment data collected from the participants will be securely saved to an encrypted mobile hard drive. Only authorised research assistants will have access to the trial data. Blood samples will be stored and processed in specialised laboratories as required.

\section{Statistical analysis}

An independent statistician who is not involved in outcome measurement will perform statistical analysis using SPSS V.24.0 software. Intention-to-treat analysis will be used for all randomised participants. Missing data will be analysed according to the likelihood estimation in the mixed effects model. Continuous data will be expressed as mean $\pm \mathrm{SD}$ or median \pm quartile interval, and enumeration data will be expressed as a relative ratio. Comparisons of baseline differences between groups will be performed using one-way analysis of variance (ANOVA) or the Kruskal-Wallis $\mathrm{H}$ test for continuous variables and $\chi^{2}$ for categorical variables. Comparisons within each group (before and after 12 weeks of intervention) will be evaluated using a paired t-test or non-parametric test. Comparisons of endpoint differences between groups will be assessed using a $2 \times 2$ ANOVA (group $\times$ time) or the Kruskal-Wallis $\mathrm{H}$ test. Multiple comparisons between the intervention arms will be performed using SNK-q test or Nemenyi test. $\mathrm{P}$ value of $<0.05$ will be considered statistically significant.

\section{Ethics and dissemination}

This trial will be conducted in accordance with the Declaration of Helsinki. Approval of this study was granted by the Ethics Committee of Yue-Yang Integrative Medicine
Hospital (Grant No. 2019-141). All participants will be fully informed of the research content and the possible disadvantages and benefits of this study, and each will sign an informed consent form before participating in the trial. Study results will be disseminated through peerreviewed publications and at academic conferences.

\section{DISCUSSION}

A variety of influencing factors may cause CI in patients with COPD, including age, disease duration, disease severity, hypercapnia, smoking and vascular dysfunction. ${ }^{45-47}$ Of those, hypoxia caused by pulmonary dysfunction is the main cause of CI in patients with COPD. The lower the partial pressure of oxygen, the higher the risk of CI. ${ }^{48} 49$ However, the pathological mechanism remains unclear. There is a hypothesis that prolonged hypoxia produces oxygen-free radicals and then releases inflammatory mediators, which affect the synthesis and metabolism of neurotransmitters and cause glial-cell activation; further damage to neurons leads to changes in brain structure and function and eventually to CI (figure 4).$^{50}{ }^{51}$ Previous studies have shown that $\mathrm{CI}$ in patients with COPD is associated with abnormal GMV. In one such study, Wang et al found that local GMV in patients with COPD was significantly reduced compared with that in the normal population. ${ }^{44}$ Yin et al found that the left-superior frontal gyrus and the right orbital part of the inferior frontal gyrus are strongly associated with pulmonary function (as measured by forced expiratory volume in one second) and cognitive function (as measured by MoCA). ${ }^{52}$ Zhang $\mathrm{H}$ et al found that the right-inferior triangular frontal gyrus in particular is related to graphic memory. ${ }^{53}$ However, the pathological mechanism of GMV atrophy in patients with COPD is very complicated and has not been conclusively demonstrated. In addition, inflammatory mediators might play an important role in the development of CI. In healthy people, higher level of the peripheral blood inflammatory mediator CRP significantly increases the risk of $\mathrm{CI},{ }^{54}$

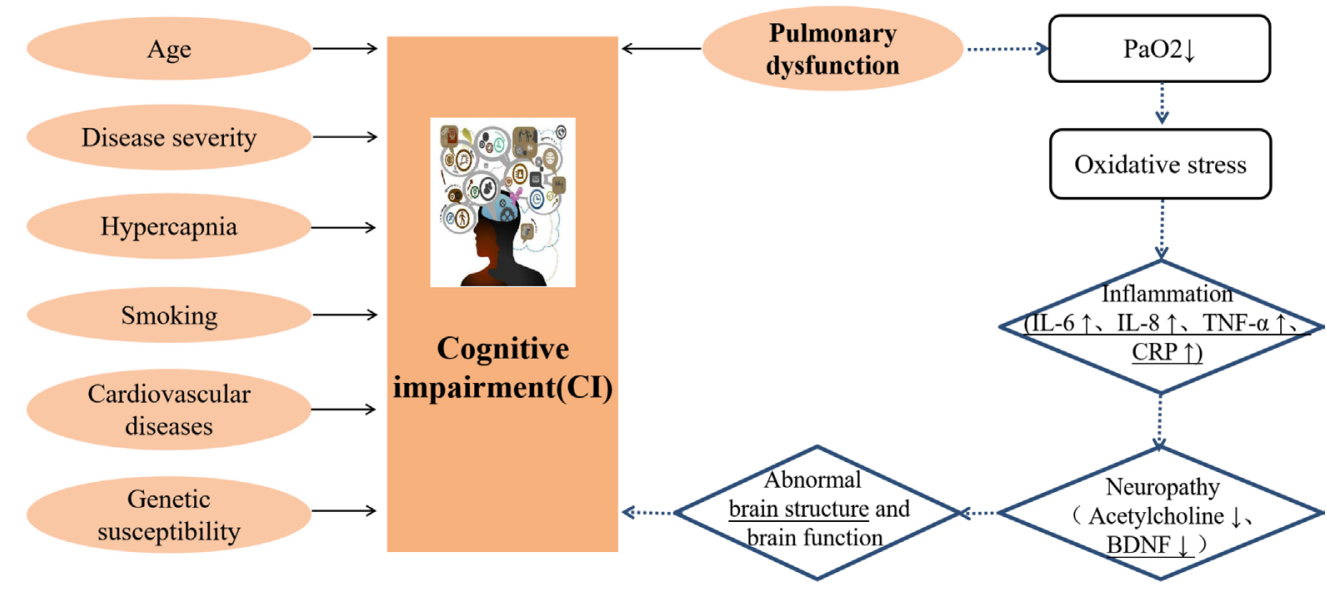

Figure 4 The possible pathological mechanisms of $\mathrm{Cl}$ in patients with COPD: pulmonary dysfunction is the main influencing factor; other influencing factors include age, disease severity, hypercapnia, smoking, cardiovascular disease and genetic susceptibility. The underlines will be observed before and after the intervention in this study. BDNF, brain-derived neurotrophic factor; CRP, C reactive protein; IL, interleukin-6; $\mathrm{PaO}_{2}$, pressure of arterial oxygen; TNF- $\alpha$, tumour necrosis factor- $\alpha$. 
especially mental-processing speed. ${ }^{55}$ In patients with Alzheimer disease, there is a meaningful correlation between inflammatory mediators and GCF. ${ }^{56}{ }^{57}$ Moreover, it has been shown that the plasma of patients with COPD often contains abnormal levels of inflammatory mediators, including IL-6, IL-8, CRP, TNF- $\alpha$ and BDNF. ${ }^{58-60}$ Therefore, in this study, we seek to observe whether PR can affect GMV and blood inflammatory mediators and to explore the possible mechanism by which exercise affects cognitive function in patients with COPD.

TCEs are moderate-intensity mind-body exercises that coordinate bodily movement, breathing, posture and attention. ${ }^{61}{ }^{62}$ Pulmonary-based Qigong exercise has strong lung-enhancing effects and is more suitable for patients with COPD. ${ }^{16-18}$ Considering that $\mathrm{CI}$ in patients with COPD is closely related to lung function impairment, this study intends to observe whether pulmonarybased Qigong exercise can improve cognitive function in such patients from the perspective of enhancing their pulmonary function.

As an important component of PR, RE can effectively improve physical function and QoL in patients with COPD. Conventional RE relies on large and expensive equipment, ${ }^{63}$ while elastic band-based RE is easily accessible and does not require much space. In addition, elastic bands can provide different levels of resistance individually. Evidence has demonstrated that elastic band-based RE has the same positive effect as conventional RE (weight machines and dumbbells) in interventions for muscle strength and QoL in patients with COPD. ${ }^{6465}$ Therefore, this study intends to observe the effect of elastic band-based RE on cognitive function in patients with COPD.

In terms of cognitive-function assessment tools, we will use the MoCA Scale and AERP P300 to assess GCF in patients with COPD. As a comprehensive, timeconsuming, easy-to-use assessment tool verified to be both sensitive and specific, the MoCA has a higher diagnostic efficacy for CI than the similar MMSE Scale. ${ }^{6667}$ In addition, it has been found that P300 latency in patients with COPD is significantly prolonged, ${ }^{35}$ and significantly differ by disease duration. ${ }^{346}$ Compared with MoCA, AERP P300 results have the clear advantage that are not affected by subjective factors. However, the specificity of AERP P300 in the diagnosis of CI is not very strong. ${ }^{69}$ Therefore, MoCA combined with AERP P300 can more accurately and objectively assess cognitive function in patients with COPD.

In summary, this article presents the study protocol for a clinical trial to investigate the effects of pulmonarybased Qigong exercise, elastic band-based RE, and a combination of both exercise on GCF, specific cognitive domains, QoL and psychological functions in patients with stable COPD. The study results will clarify the effects of $3 \mathrm{PR}$ regimens on cognitive function in patients with stable COPD and further explore possible mechanisms by which PR intervention affects cognitive function in these patients.
Trial status

This trial is currently in the recruitment phase.

Author affiliations

${ }^{1}$ School of Rehabilitation Science, Shanghai University of Traditional Chinese Medicine, Shanghai, China

${ }^{2}$ Department of Sports Medicine, Shanghai University of Sport, Shanghai, China ${ }^{3}$ Department of Respiratory Medicine, Shanghai University of Traditional Chinese Medicine Yueyang Hospital of Integrated Traditional Chinese Medicine and Western Medicine, Shanghai, China

Contributors HD and PL are joint first authors. HD, PL, ZW, HC and TW designed the study. HD drafted and organised most of the manuscript. PL drafted part of the manuscript, guided the key revision of the manuscript and participated in trial registration and ethical review. ZW contributed to the participants' recruitment. HC and TW provided design ideas for the study, collected relevant data and made substantial contributions to the production of pictures in the manuscript. XL and WW contributed to the interpretation of the results and critical revision of the manuscript for important intellectual content and approved the final version of the manuscript. All authors have read and approved the final manuscript.

Funding This study was supported by National Natural Science Foundation of China (81902307).

Competing interests None declared.

Patient consent for publication Not required.

Provenance and peer review Not commissioned; externally peer reviewed.

Supplemental material This content has been supplied by the author(s). It has not been vetted by BMJ Publishing Group Limited (BMJ) and may not have been peer-reviewed. Any opinions or recommendations discussed are solely those of the author(s) and are not endorsed by BMJ. BMJ disclaims all liability and responsibility arising from any reliance placed on the content. Where the content includes any translated material, BMJ does not warrant the accuracy and reliability of the translations (including but not limited to local regulations, clinical guidelines, terminology, drug names and drug dosages), and is not responsible for any error and/or omissions arising from translation and adaptation or otherwise.

Open access This is an open access article distributed in accordance with the Creative Commons Attribution Non Commercial (CC BY-NC 4.0) license, which permits others to distribute, remix, adapt, build upon this work non-commercially, and license their derivative works on different terms, provided the original work is properly cited, appropriate credit is given, any changes made indicated, and the use is non-commercial. See: http://creativecommons.org/licenses/by-nc/4.0/.

\section{ORCID iDs}

Hongxia Duan http://orcid.org/0000-0001-7925-8925

Xiaodan Liu http://orcid.org/0000-0002-8379-0292

\section{REFERENCES}

1 Global Initiative for Chronic Obstructive Lung Disease. Globa strategy for the diagnosis, management, and prevention of chronic obstructive pulmonary disease, 2019. Available: https://goldcopd. org/2019 [Accessed 8 Jan 2020].

2 Pelgrim CE, Peterson JD, Gosker HR, et al. Psychological comorbidities in COPD: targeting systemic inflammation, a benefit for both? Eur J Pharmacol 2019;842:99-110.

3 Lü Z, Hu P-pan, Hu X-wei, et al. [Mechanism of impaired attention network in patients with stable chronic obstructive pulmonary disease]. Zhonghua Yi Xue Za Zhi 2012;92:405-7.

4 Cleutjens FA, Franssen FM, Spruit MA, et al. Domain-Specific cognitive impairment in patients with COPD and control subjects. Int $J$ Chron Obstruct Pulmon Dis 2017;12:1-11.

5 Dogra A, Mann R, Sarkar M, et al. Pattern of cognitive dysfunction in different severity of chronic obstructive pulmonary disease. National Journal of Medical Research 2015;5:1-5.

6 Makanikas K, Andreou G, Vlachos F. Chronic obstructive pulmonary disease and semantic language abilities. J Behav Brain Sci 2019;09:130-43.

7 Yazar EE, Aydin S, Gunluoglu G, et al. Clinical effects of cognitive impairment in patients with chronic obstructive pulmonary disease. Chron Respir Dis 2018;15:306-14. 
8 Cleutjens FAHM, Spruit MA, Ponds RWHM, et al. The impact of cognitive impairment on efficacy of pulmonary rehabilitation in patients with COPD. J Am Med Dir Assoc 2017;18:420-6.

9 Troosters T, Demeyer $\mathrm{H}$, Hornikx M, et al. Pulmonary rehabilitation. Clin Chest Med 2014;35:241-9.

10 Luan $\mathrm{X}$, Tian $\mathrm{X}$, Zhang $\mathrm{H}$, et al. Exercise as a prescription for patients with various diseases. J Sport Health Sci 2019;8:422-41.

11 Desveaux L, Harrison SL, Gagnon J-F, et al. Effects of exercise training on cognition in chronic obstructive pulmonary disease: a systematic review. Respir Med 2018;139:110-6.

12 Gendron LM, Nyberg A, Saey D, et al. Active Mind-body movement therapies as an adjunct to or in comparison with pulmonary rehabilitation for people with chronic obstructive pulmonary disease. Cochrane Database Syst Rev 2018;10:CD012290.

13 Li P, Liu J, Lu Y, et al. Effects of long-term home-based Liuzijue exercise combined with clinical guidance in elderly patients with chronic obstructive pulmonary disease. Clin Interv Aging 2018;13:1391-9.

14 Liu X, Li P, Li J, et al. Home-Based prescribed pulmonary exercise in patients with stable chronic obstructive pulmonary disease. J Vis Exp 2019:59765

15 Li M. Effects of Baduanjin on cognitive function, mood and quality of life in patients with stable chronic obstructive pulmonary disease. Shenyang Sport University, 2018.

16 Ru K. Breathing regimen Liuzijue. Changchun: Jilin Science \& Technology Publishing House, 2015: 14-18.

17 Zang M, Cai GL, Lin WB, et al. Effects of the crane actions in Wuqinxi combined breathing exercise on quality of life and immune function in patients with chronic obstructive pulmonary disease]. $J$ Tradit Chin Med2017;34:819-23.

18 Wang $\mathrm{ZH}$. [Origin and principle of 'pushing up the sky to regulate the Triple Warmer' in Baduanjin].. Health Qigong2012.

19 Wang S, Yin H, Wang X, et al. Efficacy of different types of exercises on global cognition in adults with mild cognitive impairment: a network meta-analysis. Aging Clin Exp Res 2019;31:1391-400.

20 Yoon DH, Kang D, Kim H-J, et al. Effect of elastic band-based highspeed power training on cognitive function, physical performance and muscle strength in older women with mild cognitive impairment. Geriatr Gerontol Int 2017;17:765-72.

21 Hong S-G, Kim J-H, Jun T-W. Effects of 12-week resistance exercise on electroencephalogram patterns and cognitive function in the elderly with mild cognitive impairment: a randomized controlled trial. Clin J Sport Med 2018;28:500-8.

22 Aquino G, luliano E, di Cagno A, et al. Effects of combined training vs aerobic training on cognitive functions in COPD: a randomized controlled trial. Int J Chron Obstruct Pulmon Dis 2016:11:711-8.

23 Quintero AP, Bonilla-Vargas KJ, Correa-Bautista JE, et al. Acute effect of three different exercise training modalities on executive function in overweight inactive men: a secondary analysis of the BrainFit study. Physiol Behav 2018;197:22-8.

24 Timmons JF, Minnock D, Hone M, et al. Comparison of time-matched aerobic, resistance, or concurrent exercise training in older adults. Scand J Med Sci Sports 2018;28:2272-83.

25 Yang Y, XP H, Wang W, et al. Clinical study of aerobic exercise combined with resistance exercise in elderly patients with nondementia cognitive impairment after stroke]. Geriatrics \& Health Care 2018;24:265-8.

26 Song D, Yu DSF. Effects of a moderate-intensity aerobic exercise programme on the cognitive function and quality of life of community-dwelling elderly people with mild cognitive impairment: a randomised controlled trial. Int J Nurs Stud 2019;93:97-105.

27 Syed-Abdul MM, McLellan CL, Parks EJ. Effects of incremental resistance training program on quality of life in older adults. Int $J$ Exerc Sci 2017;11.

28 Brzycki M. Strength Testing-Predicting a One-Rep max from Repsto-Fatigue. J Phys Educ Recreat Dance 1993;64:88-90.

29 Thera-Band Academy. 10 myths of resistance: the truth exposed with evidence! Available: http://www.thera-bandacademy.com [Accessed 20 Aug 2020].

30 Iuliano E, di Cagno A, Aquino G, et al. Effects of different types of physical activity on the cognitive functions and attention in older people: a randomized controlled study. Exp Gerontol 2015;70:105-10.

31 O'Shea SD, Taylor NF, Paratz J. Peripheral muscle strength training in COPD: a systematic review. Chest 2004;126:903-14.

32 Miller MR, Hankinson J, Brusasco V, et al. Standardisation of spirometry. Eur Respir J 2005;26:319-38.

33 Sciriha A, Lungaro-Mifsud S, Scerri J, et al. Health status of COPD patients undergoing pulmonary rehabilitation: a comparative responsiveness of the cat and SGRQ. Chron Respir Dis 2017:14:352-9.
34 Nasreddine ZS, Phillips NA, Bédirian V, et al. The Montreal cognitive assessment, MoCA: a brief screening tool for mild cognitive impairment. J Am Geriatr Soc 2010;53:695-9.

35 Krishnamurthy S, Sivagnaname Y, Gumallapu GC. Identification of subclinical cognitive impairment in chronic obstructive pulmonary disease using auditory p300 event related potential. Monaldi Arch Chest Dis 2019;89:monaldi.2019.1039.

36 Kirkil G, Tug T, Ozel E, et al. The evaluation of cognitive functions with p300 test for chronic obstructive pulmonary disease patients in attack and stable period. Clin Neurol Neurosurg 2007;109:553-60.

37 Crișan AF, Oancea C, Timar B, et al. Cognitive impairment in chronic obstructive pulmonary disease. PLoS One 2014:9:e102468.

38 Bolló-Gasol S, Piñol-Ripoll G, Cejudo-Bolivar JC, et al. Ecological assessment of mild cognitive impairment and Alzheimer disease using the Rivermead behavioural memory test. Neurologia 2014;29:339-45.

39 JC L, Guo QH, Hong Z, et al. The role of the trailmaking test (Chinese version) in early identification of Alzheimer's disease]. Chinese Journal of Clinical Psychology 2006;14:118-20.

40 Veroff A, Snyder P, Fredrickson A, et al. P2-257: verbal fluency tests: sensitivity to disease or drug effects not affected by simplifying scoring. Alzheimer's \& Dementia 2012;8:P352.

41 Bjelland I, Dahl AA, Haug TT, et al. The validity of the hospital anxiety and depression scale. An updated literature review. J Psychosom Res 2002;52:69-77.

42 Mollayeva T, Thurairajah P, Burton K, et al. The Pittsburgh sleep quality index as a screening tool for sleep dysfunction in clinical and non-clinical samples: a systematic review and meta-analysis. Sleep Med Rev 2016;25:52-73.

43 Gong YX. Revision of Wechsler's adult intelligence scale in China. Acta psychologica sinica 1983;15:121-9.

44 Wang C, Ding Y, Shen B, et al. Altered gray matter volume in stable chronic obstructive pulmonary disease with subclinical cognitive impairment: an exploratory study. Neurotox Res 2017;31:453-63.

$45 \mathrm{Li} \mathrm{J}$, Huang Y, Fei G-H. The evaluation of cognitive impairment and relevant factors in patients with chronic obstructive pulmonary disease. Respiration 2013;85:98-105

46 Zheng G-qing, Wang Y, Wang X-tong. Chronic hypoxia-hypercapnia influences cognitive function: a possible new model of cognitive dysfunction in chronic obstructive pulmonary disease. Med Hypotheses 2008;71:111-3.

47 Pelegrino NRG, Tanni SE, Amaral RAF, et al. Effects of active smoking on airway and systemic inflammation profiles in patients with chronic obstructive pulmonary disease. Am J Med Sci 2013;345:440-5.

48 Wen X-H, Li Y, Han D, et al. The relationship between cognitive function and arterial partial pressure $\mathrm{O} 2$ in patients with COPD: a meta-analysis. Medicine 2018;97:e9599.

49 Thakur N, Blanc PD, Julian LJ, et al. Copd and cognitive impairment: the role of hypoxemia and oxygen therapy. Int $J$ Chron Obstruct Pulmon Dis 2010;5:263-9.

50 Repine JE, Bast A, Lankhorst I. Oxidative stress in chronic obstructive pulmonary disease. oxidative stress Study Group. Am J Respir Crit Care Med 1997:156:341-57.

51 Sabit R, Thomas P, Shale DJ, et al. The effects of hypoxia on markers of coagulation and systemic inflammation in patients with COPD. Chest 2010;138:47-51.

52 Yin $\mathrm{M}$, Wang $\mathrm{H}, \mathrm{Hu} \mathrm{X}$, et al. Patterns of brain structural alteration in COPD with different levels of pulmonary function impairment and its association with cognitive deficits. BMC Pulm Med 2019;19:203

53 Zhang $\mathrm{H}$, Wang $\mathrm{X}$, Lin J, et al. Reduced regional gray matter volume in patients with chronic obstructive pulmonary disease: a voxelbased morphometry study. AJNR Am J Neuroradiol 2013;34:334-9.

54 Yang J, Fan C, Pan L, et al. C-Reactive protein plays a marginal role in cognitive decline: a systematic review and meta-analysis. Int $J$ Geriatr Psychiatry 2015;30:156-65.

55 Krogh J, Benros ME, Jørgensen MB, et al. The association between depressive symptoms, cognitive function, and inflammation in major depression. Brain Behav Immun 2014;35:70-6.

56 Redwine LS, Pung MA, Wilson K, et al. Differential peripheral inflammatory factors associated with cognitive function in patients with heart failure. Neuroimmunomodulation 2018;25:146-52.

57 Janowitz D, Habes M, Toledo JB, et al. Inflammatory markers and imaging patterns of advanced brain aging in the general population. Brain Imaging Behav 2019

58 Daabis RG, Abdel Rehem RN, Hassan MM, et al. Hypogonadism in patients with chronic obstructive pulmonary disease: relationship with airflow limitation, muscle weakness and systemic inflammation. Alexandria Journal of Medicine 2016;52:27-33.

59 Shahriary A, Panahi Y, Shirali S, et al. Relationship of serum levels of interleukin 6 , interleukin 8 , and C-reactive protein with forced 
expiratory volume in first second in patients with mustard lung and chronic obstructive pulmonary diseases: systematic review and meta-analysis. Postepy Dermatol Alergol 2017;34:192-8.

60 de Araujo CLP, da Silva IRV, Reinaldo GP, et al. Pulmonary rehabilitation and BDNF levels in patients with chronic obstructive pulmonary disease: a pilot study. Respir Physiol Neurobiol 2019;259:63-9.

61 Yang KX. Premium platinum edition health Qigong book. Tianjin: Tianjin Science and Technology Press, 2014Chinese.

62 Leung RWM, McKeough ZJ, Peters MJ, et al. Short-Form Sun-style t'ai chi as an exercise training modality in people with COPD. Eur Respir J 2013;41:1051-7.

63 Alison JA, McKeough ZJ. Pulmonary rehabilitation for COPD: are programs with minimal exercise equipment effective? $J$ Thorac Dis 2014;6:1606-14.

64 Lopes JSS, Machado AF, Micheletti JK, et al. Effects of training with elastic resistance versus conventional resistance on muscular strength: a systematic review and meta-analysis. SAGE Open Med 2019;7:205031211983111.
65 Ramos EMC, de Toledo-Arruda AC, Fosco LC, et al. The effects of elastic tubing-based resistance training compared with conventional resistance training in patients with moderate chronic obstructive pulmonary disease: a randomized clinical trial. Clin Rehabil 2014;28:1096-106.

66 Villeneuve S, Pepin V, Rahayel S, et al. Mild cognitive impairment in moderate to severe COPD: a preliminary study. Chest 2012;142:1516-23.

67 JM G, Jia HG, Wang HY, et al. Evaluation of cognitive function in patients with stable chronic obstructive pulmonary disease using MoCA scale]. Journal of Hunan normal university 2016;13:99-101.

68 Reeves RR, Struve FA, Patrick G, et al. Auditory and visual p300 cognitive evoked responses in patients with COPD: relationship to degree of pulmonary impairment. Clin Electroencephalogr 1999;30:122-5.

69 Fu L, Xiang D, Subodh D, et al. Auditory p300 study in patients with convalescent bipolar depression and bipolar depression. Neuroreport 2018;29:968-73. 